CARPATHIAN J. MATH.

Volume 37 (2021), No. 3,

Pages 381 - 392
Online version at https : //www. carpathian. cunbm. utcluj. ro/

Print Edition: ISSN 1584 - 2851; Online Edition: ISSN 1843 - 4401

DOI: https://doi.org/10.37193/CJM.2021.03.02

\title{
Approximation of solutions of split equality fixed point problems with applications
}

\author{
Abubakar Adamu and Aisha A. Adam
}

\begin{abstract}
In this paper, we introduce and study an inertial algorithm for approximating solutions of split equality fixed point problem (SEFPP), involving quasi-phi-nonexpansive mappings in uniformly smooth and 2-uniformly convex real Banach spaces and establish a strong convergence theorem. We give applications of our result to split equality problem (SEP), split equality variational inclusion problem (SEVIP) and split equality equilibrium problem (SEEP). Our results extend, generalize and unify several recent inertial-type algorithms for approximating solutions of SEP and SEVIP. Moreover, to the best of our knowledge, our propose method which does not require any compactness type assumption on the operators is the first inertial algorithm for approximating solutions of SEFPP, SEP, SEVIP and SEEP in Banach spaces.
\end{abstract}

\section{INTRODUCTION}

Let $A: \mathbb{R}^{n} \rightarrow \mathbb{R}^{n}$ be a bounded linear map with adjoint $A^{*}$. In 1994, Censor and Elfving [11] introduced the following problem:

$$
\text { find } x^{*} \in C \subset \mathbb{R}^{n} \text { such that } A x^{*} \in Q \subset \mathbb{R}^{n} \text {. }
$$

Problem (1.1) is the so-called split feasibility problem (SFP) which was later used for modeling inverse problems arising from phase retrievals and in medical image reconstruction see, e.g., [24].

Remark 1.1. Observe that a solution of the SFP solves the fixed point equation

$$
x=P_{C}\left(I-\gamma A^{*}\left(I-P_{Q}\right) A\right) x, \quad x \in C,
$$

where $P_{C}$ and $P_{Q}$ are metric projections onto $C$ and $Q$, respectively, $\gamma>0$.

In 2004, Byrne [8] introduce a $C Q$ iterative algorithm to solve (1.2). His algorithm generates a sequence $\left\{x_{n}\right\}$ by

$$
x_{n+1}=P_{C}\left(I-\gamma A^{*}\left(I-P_{Q}\right) A\right) x_{n}, \quad n \geq 1,
$$

where $\gamma \in\left(0, \frac{2}{\lambda}\right)$ with $\lambda$ being the spectral radius of the operator $A^{*} A$.

Extensions and generalizations of the SFP from $\mathbb{R}^{n}$ to infinite dimensional real Hilbert spaces has been obtained by several authors (see, e.g., [26, 29] ).

In 2011, Moudafi [29] introduced a generalization of the SFP (1.1). He introduced and studied the following problem:

$$
\text { find } x^{*} \in C, y^{*} \in Q \text {, such that } A x^{*}=B y^{*} \text {, }
$$

where $H_{1}, H_{2}$ and $H_{3}$ are real Hilbert spaces, $C$ and $Q$ be nonempty closed and convex subsets of $H_{1}$ and $H_{2}$, respectively and $A: H_{1} \rightarrow H_{3}$ and $B: H_{2} \rightarrow H_{3}$ are bounded

Received: 28.04.2021. In revised form: 01.05.2021. Accepted: 03.05.2021

2010 Mathematics Subject Classification. 47H09, 47H10, 47J25 47J05, 47J20.

Key words and phrases. fixed points, quasi-phi-nonexpansive, inertia.

Corresponding author: A. Adamu; aadamu@aust.edu.ng 
linear maps. Observe that if $B=I$ (the identity map on $H_{2}$ ) and $H_{3}=H_{2}$, then problem (1.3) reduces to problem (1.1). Problem (1.3) is the so-called split equality problem (SEP). Interest in the study of the SEP stems from its usefulness in applications. For example, in decision sciences, the setting of (1.3) allows one consider agents who interplay only via some components of their decision variables (interested readers may see [5] for more on how it works). In intensity-modulated radiation therapy (IMRT), the setting of (1.3) could serve as a model that amounts to envisage a weak coupling between the vector of doses absorbed in all voxels and that of the radiation intensity (interested readers may see [10] for more on how it works).

We shall denote the solution set of problem (1.3) by

$$
\Gamma=\{x \in C, y \in Q ; A x=B y\} .
$$

Thus, $(x, y)$ solves (1.3) means that there exists $x \in C, y \in Q$ such that $A x-B y=0$. This motivates the following minimization problem:

$$
\min _{x \in C, y \in Q} \frac{1}{2}\|A x-B x\|^{2} .
$$

Motivated by these applications of problem (1.3), Moudafi [29] introduced the following alternating $C Q$ algorithm for solving problem (1.3):

$$
\left\{\begin{array}{l}
x_{n+1}=P_{C}\left(x_{n}-\gamma_{n} A^{*}\left(A x_{n}-B y_{n}\right)\right) \\
y_{n+1}=P_{Q}\left(y_{n}+\beta_{n} B^{*}\left(A x_{n+1}-B y_{n}\right)\right) .
\end{array}\right.
$$

He proved weak convergence of the sequence $\left\{\left(x_{n}, y_{n}\right)\right\}$ generated by (1.4) to a solution of (1.3).

Observe that setting $B=I$ and $\beta_{n}=1$, the second equality in algorithm (1.4) reduces to $y_{n+1}=P_{Q}\left(A x_{n+1}\right)$ and thus, the first equality gives

$$
x_{n+1}=P_{C}\left(x_{n}-\gamma_{n} A^{*}\left(A x_{n}-P_{Q}\left(A x_{n}\right)\right)\right)=P_{C}\left(x_{n}-\gamma_{n} A^{*}\left(I-P_{Q}\right) A\left(x_{n}\right)\right),
$$

which is exactly the algorithm of Bryrne [8].

Remark 1.2. Setting $C=F(T)$ and $Q=F(S)$, which are the fixed point sets of some nonexpansive type mappings, problem (1.3) reduces to the so-called split equality fixed point problem (SEFPP). That is,

$$
\text { find } x^{*} \in F(T), y^{*} \in F(S) \text { such that } A x^{*}=B y^{*} \text {. }
$$

This special case of the SEP has been studied by numerous authors in Hilbert spaces and more general Banach spaces (see, for example, $[19,22,32,36,37]$ and the references therein).

In 2018, Chidume et al. [14] introduced and studied an iterative algorithm for approximating a solution of the SEFPP (1.5) in certain Banach spaces. They proved weak convergence of the sequence generated by their algorithm and established strong convergence under semi-compactness assumption on the operators.

To dispense with the semi-compactness restriction in the theorem of Chidume et al. [14], Chidume et al. [15] introduced a CQ algorithm and proved the following strong convergence theorem:

Theorem 1.1. Let $E_{1}$ and $E_{2}$ be 2-uniformly convex and smooth real Banach spaces with dual spaces $E_{1}^{*}$ and $E_{2}^{*}$, respectively, and let $E_{3}$ be a real Banach space with dual space $E_{3}^{*}$. Let $T$ : $E_{1} \rightarrow E_{1}$ and $S: E_{2} \rightarrow E_{2}$ be closed quasi- $\phi$-nonexpansive maps with nonempty fixed point sets, 
$F(T)$ and $F(S)$, respectively. Let $A: E_{1} \rightarrow E_{3}$ and $B: E_{2} \rightarrow E_{3}$ be bounded linear maps with adjoints $A^{*}$ and $B^{*}$, respectively. Let $\left\{\left(x_{n}, y_{n}\right)\right\}$ be a sequence in $E_{1} \times E_{2}$ generated by:

$$
\left\{\begin{array}{l}
x_{1} \in E_{1}, y_{1} \in E_{2}, C_{1}=E_{1}, Q_{1}=E_{2}, e_{n} \in J_{E_{3}}\left(A x_{n}-B y_{n}\right), \\
u_{n}=J_{E_{1}}^{-1}\left(J_{E_{1}} x_{n}-\gamma A^{*} e_{n}\right), z_{n}=J_{E_{1}}^{-1}\left(\alpha J_{E_{1}} x_{n}+(1-\alpha) J_{E_{1}} T u_{n}\right), \\
v_{n}=J_{E_{2}}^{-1}\left(J_{E_{2}} y_{n}+\gamma B^{*} e_{n}\right), w_{n}=J_{E_{2}}^{-1}\left(\alpha J_{E_{2}} y_{n}+(1-\alpha) J_{E_{2}} S v_{n}\right), \\
C_{n+1}=\left\{v \in C_{n}: \phi\left(v, z_{n}\right) \leq \phi\left(v, x_{n}\right)\right\} \\
Q_{n+1}=\left\{z \in Q_{n}: \phi\left(z, w_{n}\right) \leq \phi\left(z, y_{n}\right)\right\} \\
x_{n+1}=\Pi_{C_{n+1}} x_{1}, \quad y_{n+1}=\Pi_{Q_{n+1}} y_{1} n \geq 1 .
\end{array}\right.
$$

Suppose $\Omega=\{(x, y) \in F(T) \times F(S): A x=B y\}$ is nonempty. Then $\left\{\left(x_{n}, y_{n}\right)\right\}$ converges strongly to some point $\left(x^{*}, y^{*}\right) \in \Omega$.

An inertial-type algorithm was first introduced and studied by Polyak [31], as an acceleration process in solving smooth convex minimization problems. This algorithm is a two step iterative method in which the next iterate is obtained using the previous two iterates. Numerical experiments have shown that an algorithm with inertial term requires less number of iteration than its corresponding non-inertial version. Consequently, a lot of research effort is now being put in improving existing algorithms by using inertial extrapolation (see, e.g., $[4,6,7,9,28,20,31]$ and the references contained in them).

In 2017, Li et al. [27] introduced and studied an inertial-type algorithm for approximating solutions of SEP in real Hilbert spaces. They proved the following theorem:

Theorem 1.2. Let $H_{1}, H_{2}$ and $H_{3}$ be real Hilbert spaces and let $A: H_{1} \rightarrow H_{3}$ and $B: H_{2} \rightarrow H_{3}$ be bounded linear maps with adjoints $A^{*}$ and $B^{*}$, respectively. Let $C=\left\{x \in H_{1}: c(x) \leq 0\right\}$, $Q=\left\{y \in H_{2}: q(y) \leq 0\right\}$, where $c: H_{1} \rightarrow \mathbb{R}$ and $q: H_{1} \rightarrow \mathbb{R}$ are convex and lower semicontinuous functions. Let $x_{0}, x_{1} \in H_{1}$ and $y_{0}, y_{1} \in H_{2}$ and define the sequence $\left\{\left(x_{n}, y_{n}\right)\right\} \subset$ $H_{1} \times H_{2}$ by:

$$
\left\{\begin{array}{l}
u_{n}=x_{n}+\theta_{n}\left(x_{n}-x_{n-1}\right), \\
v_{n}=y_{n}+\theta_{n}\left(y_{n}-y_{n-1}\right), \\
C_{n}=\left\{x \in H_{1}: c\left(x_{n}\right)+\left\langle\varepsilon_{n}, x-x_{n}\right\rangle \leq 0 \quad \varepsilon_{n} \in \partial c\left(x_{n}\right)\right\}, \\
Q_{n}=\left\{y \in H_{2}: q(y)+\left\langle\eta_{n}, y-y_{n}\right\rangle \leq 0 \quad \eta_{n} \in \partial q\left(y_{n}\right)\right\} \\
x_{n+1}=P_{C_{n}}\left(u_{n}-\gamma_{n} A^{*}\left(A u_{n}-B v_{n}\right)\right), \\
y_{n+1}=P_{Q_{n}}\left(v_{n}+\gamma_{n} B^{*}\left(A u_{n}-B v_{n}\right)\right),
\end{array}\right.
$$

where $\theta_{k}$ and $\gamma_{n}$ satisfy some appropriate conditions. Then the sequence $\left\{\left(x_{n}, y_{n}\right)\right\}$ converges weakly to a solution of (1.3).

Another interesting case of the SEP is the split equality variational inclusion problem (SEVIP) introduced in real Hilbert spaces by Censor et al. [12] and extended to Banach spaces by Chang et al. [13]. The setting in Banach spaces is the following: Let $E_{1}$ and $E_{2}$ be real Banach spaces with dual spaces, $E_{1}^{*}$ and $E_{2}^{*}$, respectively, and $E_{3}$ be a real Banach space. Let $M: E_{1} \rightarrow 2^{E_{1}{ }^{*}}$ and $N: E_{2} \rightarrow 2^{E_{2}{ }^{*}}$ be maximal monotone operators. Let $A: E_{1} \rightarrow E_{3}$ and $B: E_{2} \rightarrow E_{3}$ be bounded linear operators. The SEVIP is the problem of finding

$$
x \in M^{-1}(0), y \in N^{-1}(0) \text { such that } A x=B y,
$$

where $M^{-1}(0)=\left\{x \in E_{1}: 0 \in M x\right\}$ and $N^{-1}(0)=\left\{x \in E_{2}: 0 \in N x\right\}$ are called the set of zeros of $M$ and $N$, respectively. 
Recently, in 2020, Chuasuk et al. [21] introduced and studied an inertial algorithm for approximating solutions of SEVIP in real Hilbert spaces. They proved weak convergence of the sequence generated by their algorithm to a solution of the SEVIP.

\section{Our Contribution.}

- We propose an iterative method with inertial extrapolation step for solving SEFPP in real Banach spaces more general than real Hilbert spaces, and use the result to approximate solutions of split equality equilibrium problem (SEEP), split equality variational inclusion problem (SEVIP) and split equality problem (SEP).

- Our propose method does no require the assumption that $\sum \theta_{n}\left\|x_{n}-x_{n-1}\right\|<\infty$ or its alternatives imposed on $\theta_{n}$ in many inertial-type algorithms (see, e.g., $[3,4,27]$ ).

- Our propose method extends and generalizes the methods of of Li et al. [27] and Chuasuk et al. [21] to give inertial algorithms for approximating solutions of SEFPP, SEP, SEVIP and SEEP in real Banach spaces more general than real Hilbert spaces. Furthermore, unlike in [27] and [21] where weak convergence theorems were proved, we prove strong convergence theorems in the more general setting considered here.

\section{PRELIMINARIES} by

Let $E$ be a real normed space. The normalized duality map $J$ from $E$ to $2^{E^{*}}$ is defined

$$
J x:=\left\{x^{*} \in E^{*}:\left\langle x, x^{*}\right\rangle=\|x\|^{2}=\left\|x^{*}\right\|^{2}, \forall x \in E\right\} .
$$

Remark 2.3. It is well-known that if $E$ is smooth, then $J$ is single-valued and if $E$ is strictly convex, $J$ is one-to-one, and $J$ is surjective if $E$ is reflexive. Furthermore, if $E$ is uniformly smooth and uniformly convex, then the dual space $E^{*}$ is also uniformly smooth and uniformly convex and the normalized duality map $J$ and its inverse, $J^{-1}$, are both uniformly continuous on bounded sets.

Let $E$ be a smooth real Banach space and $\phi: E \times E \rightarrow \mathbb{R}$ be a mapping defined by,

$$
\phi(x, y)=\|x\|^{2}-2\langle x, J y\rangle+\|y\|^{2}, \forall x, y \in E,
$$

where $J$ is the normalized duality mapping. This function was introduced by Alber [1] and has been studied extensively by many authors (see, e.g., [2, 17, 18, 25]). It is easy to see from the definition of $\phi$ that, in a real Hilbert space $H$, equation (2.8) reduces to $\phi(x, y)=\|x-y\|^{2}, \forall x, y \in H$.

Furthermore, given $x, y \in E$, the function $\phi$ has the following properties:

$$
\begin{aligned}
& (\|x\|-\|y\|)^{2} \leq \phi(x, y) \leq(\|x\|+\|y\|)^{2} \\
& \phi(x, y) \leq\|x\|\|J x-J y\|+\|y\|\|x-y\| .
\end{aligned}
$$

Define a mapping $V: E \times E^{*} \rightarrow \mathbb{R}$ by

$$
V\left(x, x^{*}\right)=\|x\|^{2}-2\left\langle x, x^{*}\right\rangle+\left\|x^{*}\right\|^{2} .
$$

It is easy to see that

$$
V\left(x, x^{*}\right)=\phi\left(x, J^{-1} x^{*}\right), \forall x \in E, x^{*} \in E^{*} .
$$

Definition 2.1. Let $E$ be a smooth, strictly convex and reflexive real Banach space and let $C$ be a nonempty closed and convex subset of $E$. The map $\Pi_{C}: E \rightarrow C$ defined by $\tilde{x}=\Pi_{C}(x) \in C$ such that $\phi(\tilde{x}, x)=\inf _{y \in C} \phi(y, x)$ is called the generalized projection of $E$ 
onto $C$. Clearly, in a real Hilbert space $H$, the generalized projection $\Pi_{C}$ coincides with the metric projection $P_{C}$ from $H$ onto $C$.

Definition 2.2. Let $E_{1}$ and $E_{2}$ be two reflexive, strictly convex and smooth real Banach spaces. The collection of mappings $A: E_{1} \rightarrow E_{2}$ that are linear and continuous is a normed linear space with norm defined by $\|A\|=\sup _{\|x\| \leq 1}\|A x\|$. The dual operator $A^{*}: E_{2}^{*} \longrightarrow E_{1}^{*}$ defined by $\left\langle A^{*} y^{*}, x\right\rangle=\left\langle y^{*}, A x\right\rangle, \forall x \in E_{1}, y^{*} \in E_{2}^{*}$ is called the adjoint operator of $\mathrm{A}$. The adjoint operator $A^{*}$ has the property $\left\|A^{*}\right\|=\|A\|$.

Definition 2.3. Let $C$ be a nonempty closed and convex subset of a real Banach space $E$ and let $T: C \rightarrow C$ be any mapping. Then: $T$ is said to be quasi- $\phi$-nonexpansive if $F(T):=\{x \in C: T x=x\} \neq \emptyset$ and

$$
\phi(x, T y) \leq \phi(x, y) \forall x \in F(T), y \in C .
$$

Lemma 2.1 ([25]). Let $E$ be a uniformly convex and uniformly smooth real Banach space and $\left\{x_{n}\right\},\left\{y_{n}\right\}$ be sequences in $E$ such that either $\left\{x_{n}\right\}$ or $\left\{y_{n}\right\}$ is bounded. If $\lim _{n \rightarrow \infty} \phi\left(x_{n}, y_{n}\right)=0$, then, $\lim _{n \rightarrow \infty}\left\|x_{n}-y_{n}\right\|=0$.

Remark 2.4. Using inequality (2.10), it is easy to see that the converse of Lemma 2.1 is also true whenever $\left\{x_{n}\right\}$ and $\left\{y_{n}\right\}$ are both bounded.

Lemma 2.2 ([2]). Let $E$ be a reflexive, strictly convex and smooth real Banach Space with dual $E^{*}$. Then

$$
V\left(x, x^{*}\right)+2\left\langle J^{-1} x^{*}-x, y^{*}\right\rangle \leq V\left(x, x^{*}+y^{*}\right), \quad \forall x \in E, x^{*}, y^{*} \in E^{*} .
$$

Lemma 2.3 ([34]). Let $E$ be 2-uniformly convex and smooth real Banach space. Then, $J^{-1}$ is $\frac{1}{c}$-Lipschitzian from $E^{*}$ to $E$, i.e., for all $u, v \in E^{*}$, we have that

$$
\left\|J^{-1} u-J^{-1} v\right\| \leq \frac{1}{c}\|u-v\|, \text { for some } c>0 .
$$

Lemma 2.4 ([1]). Let $C$ be a nonempty closed and convex subset of a reflexive, strictly convex and smooth Banach space E. Then

$$
\phi\left(u, \Pi_{C} y\right)+\phi\left(\Pi_{C} y, y\right) \leq \phi(u, y), \forall u \in C, y \in E .
$$

Lemma 2.5 ([33]). Let $C$ be a nonempty closed and convex subset of a reflexive, strictly convex and smooth Banach space $E, A: E \rightarrow 2^{E_{1}{ }^{*}}$ be a maximal monotone operator with $A^{-1}(0) \neq \emptyset$, then for any $x \in E, y \in A^{-1}(0)$ and $r>0$ we have

$$
\phi\left(y, Q_{r}^{A} x\right)+\phi\left(Q_{r}^{A} x, x\right) \leq \phi(y, x),
$$

where $Q_{r}^{A}: E \rightarrow E$ is defined by $Q_{r}^{A} x:=(J+r A)^{-1} J x$.

\section{MAin RESUlts}

We present in this section the main results of this paper.

\subsection{Strong convergence theorem.}

Theorem 3.3. Let $E_{1}$ and $E_{2}$ be uniformly smooth and 2-uniformly convex real Banach spaces and $E_{3}$ be a uniformly smooth real Banach space. Let $A: E_{1} \rightarrow E_{3}$ and $B: E_{2} \rightarrow E_{3}$ (such that $A, B \neq 0$ ) be bounded linear operators with adjoints $A^{*}$ and $B^{*}$, respectively. Let $T: E_{1} \rightarrow E_{1}$ 
and $S: E_{2} \rightarrow E_{2}$ be closed quasi- $\phi$-nonexpansive mappings. Setting $\Gamma=\{(x, y) \in F(T) \times$ $F(S): A x=B y\}$ and assuming $\Gamma \neq \emptyset$. Let $\left\{\left(x_{n}, y_{n}\right)\right\}$ be a sequence generated by

$$
\left\{\begin{array}{l}
x_{0}, x_{1} \in E_{1}, y_{0}, y_{1} \in E_{2}, C_{1}=E_{1}, Q_{1}=E_{2}, e_{n}=J_{E_{3}}\left(A w_{n}-B t_{n}\right) \\
w_{n}=x_{n}+\alpha_{n}\left(x_{n}-x_{n-1}\right), u_{n}=J_{E_{1}}^{-1}\left(J_{E_{1}} w_{n}-\gamma A^{*} e_{n}\right) \\
r_{n}=J_{E_{1}}^{-1}\left(a_{n} J_{E_{1}} u_{n}+\left(1-a_{n}\right) J_{E_{1}} T u_{n}\right) \\
t_{n}=y_{n}+\alpha_{n}\left(y_{n}-y_{n-1}\right), v_{n}=J_{E_{2}}^{-1}\left(J_{E_{2}} t_{n}+\gamma B^{*} e_{n}\right) \\
z_{n}=J_{E_{2}}^{-1}\left(a_{n} J_{E_{2}} v_{n}+\left(1-a_{n}\right) J_{E_{2}} S v_{n}\right) \\
C_{n+1}=\left\{u \in C_{n}: \phi\left(u, r_{n}\right) \leq \phi\left(u, w_{n}\right)\right\} \\
Q_{n+1}=\left\{v \in Q_{n}: \phi\left(v, z_{n}\right) \leq \phi\left(v, t_{n}\right)\right\} \\
x_{n+1}=\Pi_{C_{n+1}} x_{1}, y_{n+1}=\Pi_{Q_{n+1}} y_{1} ; n \geq 1
\end{array}\right.
$$

where $0<a_{n}<1, \alpha \in(0,1) 0<\gamma<\frac{c}{\|A\|^{2}+\|B\|^{2}}, c=\min \left\{c_{1}, c_{2}\right\}$ where $c_{1}, c_{2}$ are constants as in Lemma 2.3. Then $\left\{\left(x_{n}, y_{n}\right)\right\}$ converges to some point $\left(x^{*}, y^{*}\right)$ in $\Gamma$.

Proof. We divide the proof into 4 steps.

Step 1. We show that $C_{n}$ and $Q_{n}$ are closed and convex for any $n \geq 1$.

Since $C_{1}=E_{1}, Q_{1}=E_{2}, C_{1}$ and $Q_{1}$ are closed and convex.

Assume $C_{n}$ and $Q_{n}$ are closed and convex for some $n \geq 1$. Since for any $(u, v) \in C_{n} \times Q_{n}$,

and

$$
\phi\left(u, r_{n}\right) \leq \phi\left(u, w_{n}\right) \Leftrightarrow 2\left\langle u, J w_{n}-J r_{n}\right\rangle \leq\left\|w_{n}\right\|^{2}-\left\|r_{n}\right\|^{2}
$$

$$
\phi\left(v, z_{n}\right) \leq \phi\left(v, t_{n}\right) \Leftrightarrow 2\left\langle v, J t_{n}-J z_{n}\right\rangle \leq\left\|t_{n}\right\|^{2}-\left\|z_{n}\right\|^{2},
$$

its easy to deduce that $C_{n+1}$ and $Q_{n+1}$ are closed and convex. Therefore, $C_{n}$ and $Q_{n}$ are closed and convex for any $n \geq 1$.

Step 2. We prove that $\Gamma \subset C_{n} \times Q_{n}$, for any $n \geq 1$. Let $K_{1}:=C_{1} \times Q_{1}$ and

$$
K_{n+1}:=\left\{(u, v) \in C_{n} \times Q_{n}: \phi\left(u, r_{n}\right)+\phi\left(v, z_{n}\right) \leq \phi\left(u, w_{n}\right)+\phi\left(v, t_{n}\right)\right\} .
$$

Then, by construction $K_{n} \subset C_{n} \times Q_{n}$.

Claim. $\Gamma \subset K_{n}$, for any $n \geq 1$. Clearly, $\Gamma \subset C_{1} \times Q_{1}$. Assume $\Gamma \subset K_{n}$ for some $n \geq 1$. Let $(x, y) \in \Gamma$, then

$$
\begin{aligned}
\phi\left(x, r_{n}\right) & =\phi\left(x, J_{E_{1}}^{-1}\left(a_{n} J_{E_{1}} u_{n}+\left(1-a_{n}\right) J_{E_{1}} T u_{n}\right)\right) \\
& =V\left(x, a_{n} J_{E_{1}} u_{n}+\left(1-a_{n}\right) J_{E_{1}} T u_{n}\right) \\
& \leq a_{n} V\left(x, J_{E_{1}} u_{n}\right)+\left(1-a_{n}\right) V\left(x, J_{E_{1}} T u_{n}\right) \\
& =a_{n} \phi\left(x, u_{n}\right)+\left(1-a_{n}\right) \phi\left(x, T u_{n}\right) \\
& \leq \phi\left(x, u_{n}\right) .
\end{aligned}
$$

By Lemma 2.2 we get

$$
\begin{aligned}
\phi\left(x, u_{n}\right) & =\phi\left(x, J_{E_{1}}^{-1}\left(J_{E_{1}} w_{n}-\gamma A^{*} J_{E_{3}}\left(A w_{n}-B t n\right)\right)\right. \\
& =V\left(x, J_{E_{1}} w_{n}-\gamma A^{*} J_{E_{3}}\left(A w_{n}-B t n\right)\right) \\
& \leq V\left(x, J_{E_{1}} w_{n}\right)-2 \gamma\left\langle J_{E_{1}}^{-1}\left(J_{E_{1}} w_{n}-\gamma A^{*} e_{n}\right)-x, A^{*} e_{n}\right\rangle \\
& =\phi\left(x, w_{n}\right)-2 \gamma\left\langle A u_{n}-A x, e_{n}\right\rangle .
\end{aligned}
$$

Thus,

$$
\phi\left(x, r_{n}\right) \leq \phi\left(x, w_{n}\right)-2 \gamma\left\langle A u_{n}-A x, e_{n}\right\rangle .
$$


Using a similar argument, we obtain that

$$
\phi\left(y, z_{n}\right) \leq \phi\left(y, t_{n}\right)-2 \gamma\left\langle B y-B v_{n}, e_{n}\right\rangle .
$$

Adding inequalities (3.14) and (3.15) and using the fact that $A x=B y$, we get

$$
\phi\left(x, r_{n}\right)+\phi\left(y, z_{n}\right) \leq \phi\left(x, w_{n}\right)+\phi\left(y, t_{n}\right)-2 \gamma\left\langle A u_{n}-B v_{n}, e_{n}\right\rangle .
$$

Using the fact that $e_{n}=J_{E_{3}}\left(A w_{n}-B t_{n}\right)$, we estimate as follows

$$
\begin{aligned}
- & 2 \gamma\left\langle A u_{n}-B v_{n}, e_{n}\right\rangle \\
= & -2 \gamma\left\|A w_{n}-B t_{n}\right\|^{2}-2 \gamma\left\langle A u_{n}-B v_{n}, e_{n}\right\rangle+2 \gamma\left\langle A w_{n}-B t_{n}, e_{n}\right\rangle \\
= & -2 \gamma\left\|A w_{n}-B t_{n}\right\|^{2}+2 \gamma\left\langle A\left(w_{n}-u_{n}\right), e_{n}\right\rangle+2 \gamma\left\langle B\left(v_{n}-t_{n}\right), e_{n}\right\rangle \\
= & -2 \gamma\left\|A w_{n}-B t_{n}\right\|^{2}+2 \gamma\left\langle J_{E_{1}}^{-1} J_{E_{1}} w_{n}-J_{E_{1}}^{-1}\left(J_{E_{1}} w_{n}-\gamma A^{*} e_{n}\right), A^{*} e_{n}\right\rangle \\
& +2 \gamma\left\langle J_{E_{2}}^{-1}\left(J_{E_{2}} t_{n}+\gamma B^{*} e_{n}\right)-J_{E_{2}}^{-1} J_{E_{2}} t_{n}, B^{*} e_{n}\right\rangle \\
\leq & \left.-2 \gamma\left\|A w_{n}-B t_{n}\right\|^{2}+\frac{2 \gamma^{2}\|A\|^{2}}{c} \|\left(A w_{n}-B t_{n}\right)\right)\left\|^{2}+\frac{2 \gamma^{2}\|B\|^{2}}{c}\right\|\left(A w_{n}-B t_{n}\right) \|^{2} \\
= & -\left(2 \gamma-\frac{2 \gamma^{2}\left(\|A\|^{2}+\|B\|^{2}\right)}{c}\right)\left\|\left(A w_{n}-B t_{n}\right)\right\|^{2} .
\end{aligned}
$$

Substituting inequality (3.17) in inequality (3.16) and using the fact that $0<\gamma<\frac{c}{\|A\|^{2}+\|B\|^{2}}$, we have that

$$
\begin{aligned}
\phi\left(x, r_{n}\right)+\phi\left(y, z_{n}\right) \leq & \phi\left(x, w_{n}\right)+\phi\left(y, t_{n}\right) \\
& -\left(2 \gamma-\frac{2 \gamma^{2}\left(\|A\|^{2}+\|B\|^{2}\right)}{c}\right)\left\|\left(A w_{n}-B t_{n}\right)\right\|^{2} \\
\leq & \phi\left(x, w_{n}\right)+\phi\left(y, t_{n}\right) .
\end{aligned}
$$

Hence $\Gamma \subset K_{n}$, for any $n \geq 1$. Thus, $\Gamma \subset C_{n} \times Q_{n}$, for any $n \geq 1$.

Step 3. We shall show that $\lim _{n \rightarrow \infty}\left(x_{n}, y_{n}\right)=\left(x^{*}, y^{*}\right) \in E_{1} \times E_{2}$.

Let $(u, v) \in \Gamma$. Since $\Gamma \subset C_{n+1} \times Q_{n+1} \subset C_{n} \times Q_{n}$ and $x_{n+1}=\Pi_{C_{n+1}} x_{1} \subset C_{n}$, then by Lemma 2.4 we have that

$$
\phi\left(x_{n}, x_{1}\right)=\phi\left(\Pi_{C_{n}} x_{1}, x_{1}\right) \leq \phi\left(u, x_{1}\right)-\phi\left(u, x_{n}\right)
$$

which implies $\left\{\phi\left(x_{n}, x_{1}\right)\right\}$ is bounded. Furthermore, $\phi\left(x_{n}, x_{1}\right) \leq \phi\left(x_{n+1}, x_{1}\right)$. Hence $\left\{\phi\left(x_{n}, x_{1}\right)\right\}$ is nondecreasing. Thus, $\lim _{n \rightarrow \infty} \phi\left(x_{n}, x_{1}\right)$ exists. By inequality (2.9), $\left\{x_{n}\right\}$ is bounded and consequently $\left\{w_{n}\right\}$ is bounded. Similarly, $\phi\left(y_{n}, y_{1}\right)$ is convergent and by inequality (2.9), $\left\{y_{n}\right\}$ is bounded and consequently $\left\{t_{n}\right\}$ is bounded.

By Lemma 2.4 we have that

$$
\phi\left(x_{m}, x_{n}\right)=\phi\left(x_{m}, \Pi_{C_{n}} x_{1}\right) \leq \phi\left(x_{m}, x_{1}\right)-\phi\left(x_{n}, x_{1}\right) \rightarrow 0, \text { as } n, m \rightarrow \infty .
$$

Hence, by Lemma 2.1 we obtain that $\left\|x_{m}-x_{n}\right\| \rightarrow 0$, as $m, n \rightarrow \infty$, which implies that $x_{n} \rightarrow x^{*} \in E_{1}$, as $n \rightarrow \infty$. Following similar argument, we also obtain that $y_{n} \rightarrow y^{*} \in E_{2}$, as $n \rightarrow \infty$.

Step 4. We show that $\left(x^{*}, y^{*}\right) \in \Gamma$.

Using the definition of $w_{n}$ and $t_{n}$, we have that 


$$
\begin{aligned}
\left\|x_{n}-w_{n}\right\| & =\left\|\alpha_{n}\left(x_{n-1}-x_{n}\right)\right\| \leq\left\|x_{n-1}-x_{n}\right\| \rightarrow 0, \text { as } n \rightarrow \infty, \\
\left\|y_{n}-t_{n}\right\| & =\left\|\alpha_{n}\left(y_{n-1}-y_{n}\right)\right\| \leq\left\|y_{n-1}-y_{n}\right\| \rightarrow 0, \text { as } n \rightarrow \infty .
\end{aligned}
$$

Since $w_{n}$ and $t_{n}$ are bounded, by Remark 2.4 we have that

$$
\lim _{n \rightarrow \infty} \phi\left(x_{n}, w_{n}\right)=0=\lim _{n \rightarrow \infty} \phi\left(y_{n}, t_{n}\right) .
$$

Since $\left(x_{n+1}, y_{n+1}\right) \in C_{n+1} \times Q_{n+1}$, we have that

$$
\begin{gathered}
\phi\left(x_{n+1}, r_{n}\right) \leq \phi\left(x_{n+1}, w_{n}\right) \rightarrow 0, \text { as } n \rightarrow \infty, \\
\phi\left(y_{n+1}, z_{n}\right) \leq \phi\left(y_{n+1}, t_{n}\right) \rightarrow 0, \text { as } n \rightarrow \infty .
\end{gathered}
$$

Therefore, $\lim _{n \rightarrow \infty} \phi\left(x_{n+1}, r_{n}\right)=0=\lim _{n \rightarrow \infty} \phi\left(y_{n+1}, z_{n}\right)$. Hence, by Lemma 2.1, we have that

$$
\lim _{n \rightarrow \infty}\left\|x_{n+1}-r_{n}\right\|=0=\lim _{n \rightarrow \infty}\left\|y_{n+1}-z_{n}\right\| \text {. }
$$

Therefore, $r_{n} \rightarrow x^{*}$. and $z_{n} \rightarrow y^{*}$. as $n \rightarrow \infty$. Let $\varepsilon=\left(2 \gamma-\frac{2 \gamma^{2}\left(\|A\|^{2}+\|B\|^{2}\right)}{c}\right)$, then from inequality (3.18) we have that

$$
\varepsilon\left\|\left(A w_{n}-B t_{n}\right)\right\|^{2} \leq \phi\left(x, w_{n}\right)+\phi\left(y, t_{n}\right)-\phi\left(x, w_{n}\right)-\phi\left(y, t_{n}\right) .
$$

This implies that

$$
\begin{aligned}
\lim _{n \rightarrow \infty}\left(\varepsilon\left\|A w_{n}-B t_{n}\right\|^{2}\right) & \leq \lim _{n \rightarrow \infty}\left(\phi\left(x, w_{n}\right)+\phi\left(y, t_{n}\right)-\phi\left(x, w_{n}\right)-\phi\left(y, t_{n}\right)\right) \\
& =\phi\left(x, x^{*}\right)+\phi\left(y, y^{*}\right)-\phi\left(x, x^{*}\right)-\phi\left(y, y^{*}\right)=0 .
\end{aligned}
$$

Using the condition on $\gamma$ we get

$$
0=\lim _{n \rightarrow \infty}\left\|A w_{n}-B t_{n}\right\|=\left\|A x^{*}-B y^{*}\right\|
$$

which implies

$$
A x^{*}=B y^{*} \text {. }
$$

Next, we show that $\left(x^{*}, y^{*}\right) \in F(T) \times F(S)$. Using Lemma 2.3 we obtain that

$$
\begin{aligned}
\left\|u_{n}-x^{*}\right\| & =\left\|J_{E_{1}}^{-1}\left(J_{E_{1}} w_{n}-\gamma A^{*} e_{n}\right)-J_{E_{1}}^{-1} J_{E_{1}} x^{*}\right\| \\
& \leq \frac{1}{c}\left\|J_{E_{1}} w_{n}-\gamma A^{*} e_{n}-J_{E_{1}} x^{*}\right\| \\
& \leq \frac{1}{c}\left(\left\|J_{E_{1}} w_{n}-J_{E_{1}} x^{*}\right\|+\gamma\|A\|\left\|A w_{n}-B t_{n}\right\|\right) .
\end{aligned}
$$

Using equation (3.19) and the fact that the normalized duality mapping $J_{E_{1}}^{-1}$ is uniformly continuous on bounded subsets of $E_{1}$, this implies that $\lim _{n \rightarrow \infty} u_{n}=x^{*}$. Also,

$$
\begin{aligned}
\left\|J_{E_{1}} r_{n}-J_{E_{1}} x^{*}\right\| & =\left\|a_{n} J_{E_{1}} u_{n}+\left(1-a_{n}\right) J_{E_{1}} T u_{n}-J_{E_{1}} x^{*}\right\| \\
& =\left\|\left(1-a_{n}\right)\left(J_{E_{1}} T u_{n}-J_{E_{1}} x^{*}\right)-a_{n}\left(J_{E_{1}} x^{*}-J_{E_{1}} u_{n}\right)\right\| \\
& \geq\left(1-a_{n}\right)\left\|J_{E_{1}} T u_{n}-J_{E_{1}} x^{*}\right\|-a_{n}\left\|J_{E_{1}} x^{*}-J_{E_{1}} u_{n}\right\|,
\end{aligned}
$$

implies that $\lim _{n \rightarrow \infty}\left\|J_{E_{1}} T u_{n}-J_{E_{1}} x^{*}\right\|=0$. By norm-to-weak continuity of $J_{E_{1}}^{-1}$, we have that $T u_{n} \rightarrow x^{*}$ as $n \rightarrow \infty$. Furthermore,

$$
\left|\left\|T u_{n}\right\|-\left\|x^{*}\right\|\right|=\left|\left\|J_{E_{1}} T u_{n}\right\|-\left\|J_{E_{1}} x^{*}\right\|\right| \leq\left\|J_{E_{1}} T u_{n}-J_{E_{1}} x^{*}\right\| \rightarrow 0 .
$$

Thus, $\lim _{n \rightarrow \infty}\left\|T u_{n}\right\|=\left\|x^{*}\right\|$. Hence, by Kadec-Klee property of $E_{1}$, we have that $\lim _{n \rightarrow \infty} T u_{n}=x^{*}$. Using this, closeness of $T$ and the fact that $\lim _{n \rightarrow \infty} u_{n}=x^{*}$, we have that 
$T x^{*}=x^{*}$. Following the same argument, we also have that $S y^{*}=y^{*}$. Thus, $\left(x^{*}, y^{*}\right) \in$ $F(T) \times F(S)$. This together with (3.20) imply that $\left(x^{*}, y^{*}\right) \in \Gamma$. This completes the proof.

Corollary 3.1. Let $E_{1}$ and $E_{2}$ be $l_{p}, L^{p}(G)$, or the Sobolev spaces $W^{p}{ }_{m}(G), 1<p \leq 2$ and $E_{3}$ be a uniformly smooth real Banach space. Let $A: E_{1} \rightarrow E_{3}$ and $B: E_{2} \rightarrow E_{3}$ (such that $A, B \neq 0$ ) be bounded linear operators with adjoints $A^{*}$ and $B^{*}$, respectively. Let $T: E_{1} \rightarrow E_{1}$ and $S: E_{2} \rightarrow E_{2}$ be closed quasi- $\phi$-nonexpansive mappings. Setting $\Gamma=\{(x, y) \in F(T) \times$ $F(S): A x=B y\}$ and assuming $\Gamma \neq \emptyset$. Let $\left\{\left(x_{n}, y_{n}\right)\right\}$ be a sequence generated by (3.11). Then $\left\{\left(x_{n}, y_{n}\right)\right\}$ converges to some point $\left(x^{*}, y^{*}\right)$ in $\Gamma$.

\section{Applications}

4.1. Split equality equilibrium problem (SEEP). Let $C$ and $Q$ be nonempty closed convex subsets of a real Banach space $E$, and let $F: C \times C \rightarrow \mathbb{R}$ be a bifunction. The equilibrium problem with respect to $F$ and $C$ is to find $z \in C$ such that

$$
F(z, y) \geq 0, \forall y \in C \text {. }
$$

The set of solutions of the equilibrium problem above is denoted by $E P(F)$. For solving the equilibrium problem, we assume that $F$ satisfies the following conditions:

(A1) $F(x, x)=0$, for all $x \in C$;

(A2) $F$ is monotone, i.e., $F(x, y)+F(y, x) \leq 0, \forall x, y \in C$;

(A3) for each $x, y, z \in C, \lim _{t \downarrow 0} F(t z+(1-t) x, y) \leq F(x, y)$;

(A4) for each $x \in C, y \longmapsto F(x, y)$ is nconvex and lower semi continuous.

The split equality equilibrium problem is finding

$$
x^{*} \in C, y^{*} \in Q \text { such that } f\left(x^{*}, x\right) \geq 0, h\left(y^{*}, y\right) \geq 0 \text { and } A x^{*}=B y^{*},
$$

for all $x \in C, y \in Q$ and $f: C \times C \rightarrow \mathbb{R} h: Q \times Q \rightarrow \mathbb{R}$ are bifunctions satisfying $\left(A_{1}\right)$ to $\left(A_{4}\right)$. We shall denote the solution set of SEEP by $\Omega$.

Lemma 4.6 ([38]). Let $E$ be a reflexive, strictly convex and uniformly smooth Banach space, and $C$ be a nonempty closed convex subset of $E$. Let $F: C \times C \rightarrow \mathbb{R}$ be a bifunction satisfying conditions (A1)-(A4), then for any $x \in E$ and $r>0$, there exists a unique point $z \in C$ such that

$$
F(z, y)+\frac{1}{r}\langle y-z, j z-j x\rangle \geq 0, \forall y \in C
$$

Lemma 4.7 ([38]). let $E$ be a reflexive, strictly convex and smooth Banach space, and $C$ be a nonempty closed convex subset of $E$. Let $F: C \times C \rightarrow \mathbb{R}$ be a bifunction satisfying conditions (A1)-(A4), then for any $x \in E$ and $r>0$, define a mapping $T_{r}: E \rightarrow C$ by:

$$
T_{r} x=\left\{x \in C: F(z, y)+\frac{1}{r}\langle y-z, j z-j x\rangle \geq 0, \forall y \in C\right\} .
$$

Then the following hold;

(1) $T_{r}$ is single-valued;

(2) $T_{r}$ is firmly nonexpansive type, i.e.

$$
\left\langle T_{r} x-T_{r} y, J T_{r} x-J T_{r} y\right\rangle \leq\left\langle T_{r} x-T_{r} y, x-y\right\rangle, \forall x, y \in E ;
$$

(3) $F\left(T_{r}\right)=E P(F)$;

(4) $E P(F)$ is closed and convex;

(5) $\phi\left(x, T_{r} u\right)+\phi\left(T_{r} u, u\right) \leq \phi(x, u), \forall x \in F\left(T_{r}\right), u \in E$. 
Theorem 4.4. Let $E_{1}$ and $E_{2}$ be uniformly smooth and 2-uniformly convex real Banach spaces and $E_{3}$ be a real Banach space. Let $C$ and $Q$ be nonempty closed and convex subsets of $E_{1}$ and $E_{2}$ respectively, and $f: C \times C \rightarrow \mathbb{R} h: Q \times Q \rightarrow \mathbb{R}$ are bifunctions satisfying $\left(A_{1}\right)$ to $\left(A_{4}\right)$ such that $\operatorname{EP}(f)$ and $\operatorname{EP}(h)$ are nonempty. Let $A: E_{1} \rightarrow E_{3}$ and $B: E_{2} \rightarrow E_{3}$ be bounded linear operators with adjoints $A^{*}$ and $B^{*}$ such that $A, B \neq 0$. Assuming that $\Omega \neq \emptyset$. Let $\left\{\left(x_{n}, y_{n}\right)\right\}$ be a sequence generated by

$$
\left\{\begin{array}{l}
x_{0}, x_{1} \in E_{1}, y_{0}, y_{1} \in E_{2}, C_{1}=E_{1}, Q_{1}=E_{2}, e_{n}=J_{E_{3}}\left(A w_{n}-B t_{n}\right) \\
w_{n}=x_{n}+\alpha_{n}\left(x_{n}-x_{n-1}\right), u_{n}=J_{E_{1}}^{-1}\left(J_{E_{1}} w_{n}-\gamma A^{*} e_{n}\right) \\
r_{n}=J_{E_{1}}^{-1}\left(a_{n} J_{E_{1}} u_{n}+\left(1-a_{n}\right) J_{E_{1}} T_{r} u_{n}\right) \\
t_{n}=y_{n}+\alpha_{n}\left(y_{n}-y_{n-1}\right), v_{n}=J_{E_{2}}^{-1}\left(J_{E_{2}} t_{n}+\gamma B^{*} e_{n}\right) \\
z_{n}=J_{E_{2}}^{-1}\left(a_{n} J_{E_{2}} v_{n}+\left(1-a_{n}\right) J_{E_{2}} S_{r} v_{n}\right) \\
C_{n+1}=\left\{u \in C_{n}: \phi\left(u, r_{n}\right) \leq \phi\left(u, w_{n}\right)\right\} \\
Q_{n+1}=\left\{v \in Q_{n}: \phi\left(v, z_{n}\right) \leq \phi\left(v, t_{n}\right)\right\} \\
x_{n+1}=\Pi_{C_{n+1}} x_{1}, y_{n+1}=\Pi_{Q_{n+1}} y_{1} ; n \geq 1
\end{array}\right.
$$

where $T_{r} x=\left\{z \in C: f(z, y)+\frac{1}{r}\left\langle y-z, J_{E_{1}} z-J_{E_{1}} x\right\rangle \geq 0, \forall y \in C\right\}, x \in E_{1}, S_{r} v=$ $\left\{w \in Q: h(w, y)+\frac{1}{r}\left\langle y-w, J_{E_{2}} w-J_{E_{2}} v\right\rangle \geq 0, \forall y \in Q\right\}, v \in E_{2}, r>0,0<a_{n}<1$, $\alpha \in(0,1), 0<\gamma<\frac{c}{\|A\|^{2}+\|B\|^{2}}, c=\min \left\{c_{1}, c_{2}\right\}$, where $c_{1}, c_{2}$ are constants as in Lemma 2.3. Then $\left\{\left(x_{n}, y_{n}\right)\right\}$ converges to some point $\left(x^{*}, y^{*}\right)$ in $\Omega$.

Proof. Setting $T=T_{r}$ and $S=S_{r}$, from Lemma 4.7(3) and (5) we have that $S$ and $T$ are quasi- $\phi$-nonexpansive. And by Lemma 4.7(3) and Theorem 3.3 we get the desired result.

\subsection{Split Equality Variational Inclusion Problem (SEVIP).}

Theorem 4.5. Let $E_{1}$ and $E_{2}$ be uniformly smooth and 2-uniformly convex real Banach spaces and $E_{3}$ be a real Banach space. Let $M: E_{1} \rightarrow 2^{E_{1}^{*}}$ and $N: E_{2} \rightarrow 2^{E_{2}^{*}}$ be maximal montone operators such that $M^{-1}(0)$ and $N^{-1}(0)$ are nonempty. Let $A: E_{1} \rightarrow E_{3}$ and $B: E_{2} \rightarrow E_{3}$ be bounded linear operators with adjoints $A^{*}$ and $B^{*}$ such that $A, B \neq 0$. Assuming that $\Omega=$ $\left\{(x, y) \in M^{-1}(0) \times N^{-1}(0): A x=B y\right\} \neq \emptyset$. Let $\left\{\left(x_{n}, y_{n}\right)\right\}$ be a sequence generated by

$$
\left\{\begin{array}{l}
x_{0}, x_{1} \in E_{1}, y_{0}, y_{1} \in E_{2}, e_{n}=J_{E_{3}}\left(A w_{n}-B t_{n}\right) \\
w_{n}=x_{n}+\alpha_{n}\left(x_{n}-x_{n-1}\right), u_{n}=J_{E_{1}}^{-1}\left(J_{E_{1}} w_{n}-\gamma A^{*} e_{n}\right) \\
r_{n}=J_{E_{1}}^{-1}\left(a_{n} J_{E_{1}} u_{n}+\left(1-a_{n}\right) J_{E_{1}} Q_{r}^{M} u_{n}\right) \\
t_{n}=y_{n}+\alpha_{n}\left(y_{n}-y_{n-1}\right), v_{n}=J_{E_{2}}^{-1}\left(J_{E_{2}} t_{n}+\gamma B^{*} e_{n}\right) \\
z_{n}=J_{E_{2}}^{-1}\left(a_{n} J_{E_{2}} v_{n}+\left(1-a_{n}\right) J_{E_{2}} Q_{r}^{N} v_{n}\right) \\
C_{n+1}=\left\{u \in C_{n}: \phi\left(u, r_{n}\right) \leq \phi\left(u, w_{n}\right)\right\} \\
Q_{n+1}=\left\{v \in Q_{n}: \phi\left(v, z_{n}\right) \leq \phi\left(v, t_{n}\right)\right\} \\
x_{n+1}=\Pi_{C_{n+1}} x_{1}, y_{n+1}=\Pi_{Q_{n+1}} y_{1} ; n \geq 1
\end{array}\right.
$$

where $Q_{r}^{M}=\left(J_{E_{1}}+r M\right)^{-1} J_{E_{1}}, Q_{r}^{N}=\left(J_{E_{2}}+r N\right)^{-1} J_{E_{2}}, 0<a_{n}<1, \alpha \in(0,1)$, $0<\gamma<\frac{c}{\|A\|^{2}+\|B\|^{2}}, c=\min \left\{c_{1}, c_{2}\right\}$, where $c_{1}, c_{2}$ are constants as in Lemma 2.3. Then $\left\{\left(x_{n}, y_{n}\right)\right\}$ converges to some point $\left(x^{*}, y^{*}\right)$ in $\Omega$.

Proof. Setting $T=Q_{r}^{M}$ and $S=Q_{r}^{N}$, from Lemma 2.5 we have that $Q_{r}^{M}$ and $Q_{r}^{N}$ are quasi- $\phi$-nonexpansive. And by Theorem 3.3 we get the desired result. 


\subsection{Split equality problem. The split equality problem is to find}

$$
x \in c, y \in Q \text { such that } A x=B y \text {. }
$$

Theorem 4.6. Let $E_{1}$ and $E_{2}$ be uniformly smooth and 2-uniformly convex real Banach spaces and $E_{3}$ be a real Banach space. Let $A: E_{1} \rightarrow E_{3}$ and $B: E_{2} \rightarrow E_{3}$ be bounded linear operators with adjoints $A^{*}$ and $B^{*}$ such that $A, B \neq 0$. Assuming $\Gamma \neq \emptyset$. Let $\left\{\left(x_{n}, y_{n}\right)\right\}$ be a sequence generated by

$$
\left\{\begin{array}{l}
x_{0}, x_{1} \in E_{1}, y_{0}, y_{1} \in E_{2}, C_{1}=E_{1}, Q_{1}=E_{2}, e_{n}=J_{E_{3}}\left(A w_{n}-B t_{n}\right) \\
w_{n}=x_{n}+\alpha_{n}\left(x_{n}-x_{n-1}\right), u_{n}=J_{E_{1}}^{-1}\left(J_{E_{1}} w_{n}-\gamma A^{*} e_{n}\right) \\
r_{n}=J_{E_{1}}^{-1}\left(a_{n} J_{E_{1}} u_{n}+\left(1-a_{n}\right) J_{E_{1}} \Pi_{C} u_{n}\right) \\
t_{n}=y_{n}+\alpha_{n}\left(y_{n}-y_{n-1}\right), v_{n}=J_{E_{2}}^{-1}\left(J_{E_{2}} t_{n}+\gamma B^{*} e_{n}\right) \\
z_{n}=J_{E_{2}}^{-1}\left(a_{n} J_{E_{2}} v_{n}+\left(1-a_{n}\right) J_{E_{2}} \Pi_{Q} v_{n}\right) \\
C_{n+1}=\left\{u \in C_{n}: \phi\left(u, r_{n}\right) \leq \phi\left(u, w_{n}\right)\right\} \\
Q_{n+1}=\left\{v \in Q_{n}: \phi\left(v, z_{n}\right) \leq \phi\left(v, t_{n}\right)\right\} \\
x_{n+1}=\Pi_{C_{n+1}} x_{1}, y_{n+1}=\Pi_{Q_{n+1}} y_{1} ; n \geq 1
\end{array}\right.
$$

where $0<a_{n}<1, \alpha \in(0,1), 0<\gamma<\frac{c}{\|A\|^{2}+\|B\|^{2}}, c=\min \left\{c_{1}, c_{2}\right\}$, where $c_{1}, c_{2}$ are constants as in Lemma 2.3. Then $\left\{\left(x_{n}, y_{n}\right)\right\}$ converges to some point $\left(x^{*}, y^{*}\right)$ in $\Gamma$.

Proof. Setting $T=\Pi_{C}$ and $S=\Pi_{Q}$, from Lemma 2.4 we have that $\Pi_{C}$ and $\Pi_{Q}$ are quasi$\phi$-nonexpansive. And by Theorem 3.3 we get the desired result.

Acknowledgments. The authors appreciate the support of their institution and the African Developement Bank. Moreover, this research was completed during Dr. Abubakar Adamu's post doctoral research fellowship at AUST.

\section{REFERENCES}

[1] Alber, Ya. Metric and generalized projection operators in Banach spaces: properties and applications. In: Kartsatos, A. G. (ed.) Theory and applications of nonlinear operators of accretive and monotone type. Marcel Dekker, New York, pp. 15-50 (1996).

[2] Alber, Ya.; Ryazantseva, I. Nonlinear Ill Posed Problems of Monotone Type, Springer, London (2006).

[3] Alvarez, F. Weak convergence of a relaxed and inertial hybrid projection-proximal point algorithm for maximal monotone operators in Hilbert space. SIAM J. Optim 14 (2004), no. 3, 773-782.

[4] Alvarez, F.; Attouch, H. An inertial proximal method for maximal monotone operators via discretization of nonlinear oscillator with damping. Set-valued Anal. 9 (2001) no. 1-2, 2-11.

[5] Attouch, H.; Bolte, J.; Redont, P.; Soubeyran, A. Alternating proximal algorithms for weakly coupled minimization problems. Applications to dynamical games and PDEs. J. Convex Analysis. 15 (2008), 485-506.

[6] Bot, R. I.; Csetnek, E. R.; Hendrich, C. Inertial Douglas-Rachford splitting for monotone inclusion problems. Appl. Math. Comput. 256 (2015), 472-487.

[7] Beck, A.; Teboulle, M. A fast iterative shrinkage-thresholding algorithm for linear inverse problem. SIAM J. Imaging Sci. 2 (2009), no. 1, 183-202.

[8] Byrne, C. Iterative oblique projection onto convex sets and the split feasibility problem. Inverse Problems 18 (2002), 441-453

[9] Chan, R. H.; Ma, S.; Yang, J. F. Inertial proximal ADMM for linearly constrained separable convex optimization. SIAM J. Imaging Sci. 8 (2015), no. 4, 2239-2267.

[10] Censor, Y.; Bortfeld, T.; Martin, B.; Trofimov, A. A unified approach for inversion problems in intensitymodulated radiation therapy. Physics in Medicine and Biology. 51 (2006), 2353-2365.

[11] Censor, Y.; Elfving, T. A multiprojection algorithm using Bregman projections in a product space. Numer. Algorithms 8 (1994), no. 2-4, 221-239.

[12] Censor, Y.; Gibali, A.; Reich, S. Algorithms for the split variational inequality problem. Numer. Algorithms 59 (2012), no. 2, 301-323

[13] Chang, S. S.; Wang, L.; Qin, L.; Ma, Z. Strongly convergent iterative methods for split equality variational inclusion problems in Banach spaces. Acta Math. Sci. B 36 (2016), no. 6, 1641-1650. 
[14] Chidume, C. E.; Romanus, O. M.; Nnyaba, U. V. An iterative algorithm for solving split equality fixed point problems for a class of nonexpansive-type mappings in Banach spaces. Numer. Algorithms 82 (2019), no. 3, 987-1007

[15] Chidume, C. E.; Romanus, O. M.; Nnyaba, U. V. An iterative algorithm for solving split equilibrium problems and split equality variational inclusions for a class of nonexpansive-type maps Optimization 67 (2018), no. 11, 1949-1962.

[16] Chidume, C. E.; Ikechukwu, S. I.; Adamu, A. Inertial algorithm for approximating a common fixed point for a countable family of relatively nonexpansive maps. Fixed Point Theory Appl. 2018, Paper No. 9, 9 pp.

[17] Chidume, C. E.; Adamu, A; Chinwendu, L. O. A Krasnoselskii-type algorithm for approximating solutions of variational inequality problems and convex feasibility problems. J. Nonlinear Var. Anal. 2(2018) no. 2 203-218.

[18] Chidume, C. E.; Chinwendu, L. O.; Adamu, A. A hybrid algorithm for approximating solutions of a variational inequality problem and a convex feasibility problem. Advances in Nonlinear Variational Inequalities 21 (2018), no. 1, 46-64.

[19] Chidume, C. E.; Ndambomve, P.; Bello, A. U. The split equality fixed point problem for demicontractive mappingpings. J. Non. Ana. Optim 6 (2015), no. 1, 61-69.

[20] Dong, Q. L.; Yuan, H. B.; Je, C. Y.; Rassias, Th. M. Modified inertial Mann algorithm and inertial CQalgorithm for nonexpansive mappings. Optim. Lett. 12 (2018), 87-102. https:/ /doi.org/10.1007/s11590-0161102-9.

[21] Chuasuk, P.; Ogbuisi, F,; Shehu, Y.; Cholamjiak, P. New inertial method for generalized split variational inclusion problems. Journal of Industrial and Management Optimization 13 (5) DOI: 10.3934/jimo.2020123

[22] Giang, D. M.; Strodiot, J. J.; Nguyen, V. H. Strong convergence of an iterative method for solving the multiple-set split equality fixed point problem in a real Hilbert space. Rev. R. Acad. Cienc. Exactas Fís. Nat. Ser. A Mat. RACSAM 111 (2017), no. 4, 983-998.

[23] Gossez, J. P.; Lami-Dozo, E. Some geometric properties related to the fixed point theory for nonexpansive mappings. Pacific J. Math. 40 (1972), 365-573.

[24] Hurt, N. E. Phase Retrieval and Zero Crossings: Mathematical Methods in Image Reconstruction (Kluwer Academic, Dordrecht, The Netherlands, 1989).

[25] Kamimura, S.; Takahashi, W. Strong convergence of a proximal-type algorithm in a Banach space SIAM J. Optim. 13 (2002), 938-945.

[26] Kraikaew, R.; Saejung, S. On split common fixed point problems. J. Math. Anal. Appl. 415 (2014), 513-524.

[27] Li, M. X.; Kao, X. P.; Che, H. T. Relaxed inertial accelerated algorithms for solving split equality feasibility problem. J. Nonlinear Sci. Appl. 10 (2017), 4109-4121

[28] Lorenz, D. A.; Pock, T. An inertialforward-backward algorithm for monotone inclusions. J. Math. Imaging Vis. 51(2015), no. 2 311-325.

[29] Moudafi, A., Alternating CQ-algorithm for convex feasibility and split fixed-point problems. J. Nonlinear Convex Anal. 15 (2014), no. 4, 809-818.

[30] Opial, Z. O. Weak convergence of the sequence of successive approximations for nonexpansivemappings. Bull. Amer. Math. Soc. 73 (1967), 591-597.

[31] Polyak, B. T. Some methods of speeding up the convergence of iteration methods. USSR Comput. Math. Math. Phys. 4 (1964), no. 5, 1-17.

[32] Wu, Y. J.; Chen, R. D.; Shi, L. Y. Split equality problem and multiple-sets split equality problem for quasinonexpansive multi-valued mappings. J. Inequal. Appl. 2014 (2014), 8 pp.

[33] Wei, L.; Zhou, H. Y. The new iterative scheme with errors of zero point for maximal operator in Banach space. Math. Appl. 19 (2006), no. 1, 101-105. (in Chinese)

[34] Xu, H. K. Inequalities in Banach spaces with applications. Nonlinear Anal. 16 (1991), no. 12, 1127-1138.

[35] Censor, Y.; Segal, A. The split common fixed point problem for directed operators. J. Conv. Anal. 16 (2009), 587-600.

[36] Zhao, J.; Zong, H.; Liu, G.; Zhang, H. Solving variational inequality and split equality common fixed-point problem without prior knowledge of operator norms. J. Nonlinear Sci. Appl. 9 (2016), 5428-5440

[37] Zhao, J. Solving split equality fixed-point problem of quasi-nonexpansive mappings without prior knowledge of operators norms. Optimization 64 (2015), no. 12, 2619-2630.

[38] Zhang, S. S. Generalized mixed equilibrium problem in Banach spaces. Appl. Math. Mech. (English Ed.) 30 (2009), no. 9, 1105-1112.

\author{
AFricAN University OF SCIENCE AND TECHNOLOGY, ABUJA \\ KM 10 AIRPORT ROAD, FCT, GALADIMAWA, NIGERIA \\ Email address: aadamu@aust. edu.ng \\ Email address: aaminu@aust.edu.ng
}

